\title{
Resümee und Ausblick: Serien der Zukunft - Familien der Zukunft
}

Im März 2020 titelt der konservative Journalist David Brooks in The Atlantic „The Nuclear Family Was a Mistake“ (vgl. Brooks 2020). In seinem Artikel beklagt Brooks, dass die Kernfamilie mehr und mehr an gesellschaftlicher Funktionalität verlöre und als Struktur überlastet sei. Die US-amerikanische Gesellschaft habe sich gewandelt, Scheidungsraten seien gestiegen, Frauen seien werktätig, alleinerziehende Eltern immer häufiger. Auch wenn die Familie in den 1950er und 1960er Jahren tatsächlich stabil gewesen sei, sei das Bild der Kernfamilie heute nicht mehr repräsentativ bzw. erreichbar für die deutliche Mehrheit der US-amerikanischen Bevölkerung. Brooks größter Kritikpunkt ist dabei die Vereinsamung des Individuums. Diese Isolation sei im Modell der Kernfamilie fest verankert: „A code of family self-sufficiency prevails: Mom, Dad, and the kids are on their own, with a barrier around their island home." (Brooks 2020). Entsprechende Isolation, auch im Alter, habe es in der Groß- und erweiterten Familie früherer Zeiten nicht gegeben. Diese habe wie ein soziales und emotionales Auffangbecken in allen Lebenslagen funktioniert. Die Kernfamilie sei somit ein Privileg der Mittel- und Oberschicht, die sich entsprechende Absicherungen, d. h. Therapie, Nachhilfe, Freizeitaktivitäten etc., leisten könne. Die Mehrheit der US-Familien lebe bereits in anderen Familienformen. Um dieser Realität gerecht zu werden, fordert Brooks die Abkehr vom Ideal der Kernfamilie hin zu erweiterten Familien (,extendend and forged families“) - egal, ob basierend auf Blutsoder Wahlverwandtschaft -, nach denen ein gesellschaftliches Bedürfnis bestehe (vgl. Brooks 2020). In Bezugnahme auf Brooks konstatiert die Journalistin Judith Luig in der ZEIT, dass auch in Deutschland das Ideal der Kernfamilie immer unerreichbarer und in der Realität durch andere Lebensformen, z. B. alleinerziehende Eltern, Patchwork-Familien oder Ein bis Zwei-Personen-Haushalte, ersetzt werde. Ebenso wie Brooks fordert sie ein Umdenken in eine neue Richtung (vgl. Luig 2020). 
In Anbetracht der Aussagen Brooks und Luigs, sowie der Untersuchungsergebnisse dieser Arbeit, welche der Video-on-Demand-Serie einen konservativen Kern im progressiven Gewand und den permanenten Bezug auf den idealisierten Mythos der Kernfamilie bescheinigen und vor allem von einem übergeordneten soziologischen Standpunkt aus wäre es von großem Interesse, einen Vergleich zwischen der medialen Repräsentation der Familie und den prominenten gesellschaftlichen Diskursen anzustellen und zu erörtern, inwieweit die Darstellungsformen der Video-on-Demand-Serie und die dort dominanten Weltmodelle und Idealvorstellungen mit der Realität übereinstimmen. Allerdings kann dies im gegebenen Rahmen nicht geschehen, ohne ins Fachfremde oder Spekulative abzugleiten. Die vorliegende Untersuchung kann lediglich die Basis für einen solchen Vergleich bilden. Dementsprechend kann nicht beantwortet werden, inwiefern eine gesellschaftliche Neuorientierung, wie sie Brooks und Luig fordern, bereits stattfindet. Sehr wohl feststellen lässt sich, dass sich in der Familienrepräsentation der Video-on-Demand-Serie kein entsprechender Wandel fort von der Kernfamilie hin zur erweiterten Familie vollzieht. Mögen die Entwicklungen, welche im Vergleich zu den Serien der 1950er und 1960er Jahre stattgefunden haben, auch radikal erscheinen, so handelt es sich doch um oberflächliche Veränderungen. Insbesondere die Darstellungsweise der Charaktere und Problematiken ist vielschichtiger und ambivalenter als zuvor, eine Konsistenz der Weltmodelle kann nicht ohne Weiteres vollständig hergestellt werden. Dies lädt dazu ein, diese Serien als komplexer als ihre Vorgänger wahrzunehmen und ihnen somit eine höhere Qualität zuzusprechen. Dieser vermeintliche Anstieg der Komplexität lässt - bei genauerer Betrachtung - umso deutlicher hervortreten, dass die grundlegende Aussage der Texte, die vermittelten Normen und Werte, Weltbilder und Gesellschaftsverständnisse in ihrem Kern konstant bleiben: So erweist sich das Modell der nach außen abgegrenzten und sich selbst genügenden Kernfamilie - eben das Modell, welches Brooks als nicht mehr zeitgemäß angreift - als äußerst persistent. Durch Brooks Kritik, die keinen Bezug auf die konkrete mediale Tradierung dieses Ideals nimmt, sondern dessen realweltliche, soziale Aspekte fokussiert, wird deutlich, dass es sich hier nicht um eine auf TV-Serien begrenzte, sondern auch um eine Idealvorstellung mit konkreter Auswirkung auf die (US-amerikanische) Lebensrealität handelt.

Die Beständigkeit des Mythos begründet sich in seiner tiefen Verankerung im kulturellen Wissen: Erlerntes und durch Prägung Verinnerlichtes wird perpetuiert. Vor allem aber hat sich das attraktive Grundversprechen des Modells der Kernfamilie seit den 1950er Jahren nicht geändert. Stabilität und Überschaubarkeit in einer chaotischen Welt. Ein eindeutiger Rahmen, klare und geordnete 
Strukturen und eine - wenn auch konservative, so doch - funktionierende Hierarchie erscheinen als ein einfacher Zugang zu einem möglichst sorgenfreien Leben. Diese enge und medial gefestigte Verbindung des Kernfamilienideals mit stabilem und sicherem Leben lässt andere Modelle menschlichen Zusammenlebens in den Hintergrund treten. Insbesondere angesichts aktueller gesellschaftlicher Entwicklungen in den USA kann die Attraktivität dieses Grundversprechens erklärt werden: Auch befeuert durch die Regierungsperiode Trump schwindet das Vertrauen der US-Amerikaner in staatliche Institutionen. Libertäres Gedankengut erfährt einen Aufschwung, gesellschaftliche Fronten verhärten sich und der ohnehin populäre Gedanke, des Individuums, das auf eigene Faust für sein Recht kämpfen muss gewinnt weiter an Bedeutung. Im Gegensatz zu den bedrohlichen, als übergriffig wahrgenommenen staatlichen Institutionen und einer gespaltenen Gesellschaft, in der das Individuum keine Rolle mehr findet, steht das Überschaubare, Selbstbestimmte und Bekannte der Familie. Aus einem Sicherheitsbedürfnis heraus wird diese als unwandelbar und stabil dargestellt. Durch Biologisierung, deren Darstellung als Stamm oder Rudel wird sie zu einem unveränderbaren Faktum erhoben.

An dieser Stelle lässt sich auf die Überlegungen des Soziologen Michel Maffesolis zum sogenannten ,postmodernen Nomadentum“ (Maffesoli 2014: 107) und des „Neo-Tribalismus“ (Keller 2006 a): 217) verweisen: Maffesoli beobachtet, dass abstrakte staatliche Autoritäten, sowie zentrale, kollektivierende Leitbilder in der postmodernen Gesellschaft an Wirkmacht verlieren und das diesbezügliche Vertrauens- und Sicherheitsgefühl schwindet (vgl. Keller 2006 b): $110 \mathrm{ff}$.). An die Stelle der Suche nach einer eigenen Identität, eines Lebens nach einem bestimmten Arbeitsethos oder einer zentralen politischen Linie und einem distanzierten Umgang mit Anderen, tritt ein hedonistisches Dasein und die temporäre, distanzlose Identifikation mit „Stämmen“, d. h. mit Systemen, die gleiche Erfahrungen teilen (vgl. Keller 2006 a): 214, 217, vgl. Keller 2006 b): 114). In der Postmoderne, deren Produkte auch aktuelle Familiendarstellungen sind, wird moderner Rationalismus durch Leidenschaftliches, Nicht-Rationales abgelöst. Weniger mechanische, als vielmehr organische Strukturen stehen nunmehr im Vordergrund. Die Gesellschaft zersplittert in eine Vielzahl von Stämmen mit unterschiedlichen Werten. Auch zwischen den bestehenden Systemen bzw. Stämmen treibt das Individuum allerdings weiter umher, bleibt ein „Fremder unter Fremden“, hundertprozentige Sicherheit gibt es nicht (vgl. Keller 2006 a): 217, vgl. Keller 2006 b): 107). „Strukturell und existenziell gesehen ist in diesem Verständnis jeder und jede ein»rolling stone«, eine dahintreibende vagabundierende Figur und multiple Person im»Abenteuer der Existenz«" (Keller 2006 b): 121). Gerade dadurch, dass Sie mit Familien immer gleiche, unwandelbare Systeme 
darstellen, die im Gegensatz zum allgegenwärtigen „Umherirren“ („l'errance“, Maffesoli 1997: 26) und zur „Vergänglichkeit der Dinge“ (,l'impermanence des choses“, Maffesoli 1997: 26) stehen, werden bestimme mediale Formate so attraktiv: Sie stiften Sicherheit, präsentieren das Leben in einem „Stamm“, der nicht verlassen werden muss. Aus diesem Sicherheitsbedürfnis heraus wird das bestehende Modell immer weiter gefestigt und durch Wiederholung zementiert. Die teilweise archaisierenden und emotionalen Strategien, mit denen die Serien ihre Weltbilder legitimieren, fügen sich ebenso in Maffesolis Blick auf die Postmoderne ein, die durch „Rückkehr zum Archaismus“ und einen Bedeutungsverlust des „Rationalismus“ gekennzeichnet ist (Keller 2004: 364). Die Video-on-Demand Serie ist somit ein exemplarischer Vertreter der Postmoderne im Sinne Maffesolis.

Die vordergründige Intention mancher Video-on-Demand-Serien ist es durchaus, das Bild einer möglichst vielfältigen und heterogenen Gesellschaft zu vermitteln und bisher marginalisierte oder als abweichend und negativ konnotierte Gruppen auf positive Weise in die Weltmodelle zu integrieren. Durch die beständige Rückkehr zur Heteronormativität, dem Beharren auf dem Mythos und der Anpassung alles Heterogenen handelt es sich jedoch nicht um eine tatsächliche Vielfalt, sondern vielmehr um eine Schein-Heterogenität. Treffen alternative Formen des Zusammenlebens innerhalb einer Serie auf die Kernfamilie, so ist deren Abwertung - aus analytischer Perspektive - und eine damit einhergehende Aufwertung des Ideals gewiss. Eigentlich vielfältigen Zusammenhängen und Gegebenheiten setzen die Video-on-Demand-Serien meines Analysekorpus ein Weltmodell entgegen, das auf den ersten Blick vielleicht komplex und ambivalent erscheint, dessen normativer Kern - das Festhalten an der traditionellen Kernfamilie - aber sehr eindimensional ist.

Bieten Serien außerhalb des Video-on-Demand-Bereiches alternative Inhalte und Konzeptionen? Durchaus gibt es Produktionen, die andere Formen des Zusammenlebens inszenieren, ohne dabei eine negative Abweichung von der Kernfamilie zu betonen. Teilweise werden diese Serien sogar über die Plattformen der Video-on-Demand-Anbieter distribuiert, jedoch ohne von diesen produziert worden zu sein. Die schwedische Fernsehserie Bonusfamiljen, die in Deutschland zuerst auf Netflix unter dem Titel Die Patchworkfamilie veröffentlicht wurde, zeigt das Zusammenleben in einem größeren familiären Verband, der durch diverse Scheidungen und Heiraten zustande kam. Obwohl hier ähnliche Problematiken wie in kernfamiliären Verbänden auftreten, entwickeln sich darüber hinaus gehende Dynamiken wie die Beziehung zwischen Stiefgeschwistern oder zwischen Ehemann und Ex-Ehemann. Ebenso wird die Großelterngeneration zentral 
miteinbezogen. Auch US-amerikanische Serien warten mit Familienkonstellationen auf, die nicht in jedem Fall einem kernfamiliären und heteronormativen Ideal angeglichen werden. Die Serie Single Parents beispielsweise setzt sich mit dem Leben mehrerer alleinerziehender Eltern auseinander. Die Serie Parenthood inszeniert nicht nur das Leben einer Großfamilie, sondern lässt auch von der traditionellen Norm abweichende Rollenbilder wie das des Hausmannes zu. Protagonistin der Serie Mom ist die alleinerziehende und ehemals alkohol- und drogenabhängige Christy. Mom fokussiert den Konflikt zwischen drei Generationen von Müttern - Christy, ihrer Mutter und ihrer Tochter. Alle drei Figuren sind jung Mutter geworden, ohne jedoch einen verlässlichen Partner an ihrer Seite zu haben. Christys Tochter wird gleich zu Beginn der Serie schwanger (vgl. Mom I/2: 0:00). Somit wird die Thematik der alleinerziehenden Mutterschaft aus drei Perspektiven in den Blick genommen. Problematische Situationen werden hier weniger in einem familiären Verband gelöst als vielmehr durch den Zusammenhalt der drei Mütter (vgl. Mom I/2: 18:20). Auch wenn die gezeigte Familienform nicht als ideal inszeniert wird - sowohl Christy als auch ihre Mutter müssen stets mit ihrer Suchtproblematik und zahlreichen weiteren Problemen kämpfen (vgl. Mom I/1: 09:00) -, so liegt diese spezielle Art von Familie und eben nicht das rasche Streben nach einer traditionellen Familie der Serie konstitutiv zugrunde.

Ebenso stehen in Life in Pieces (in Deutschland auf Prime Video verfügbar) und Modern Family (in Deutschland auf Netflix verfügbar) Großfamilien und die unterschiedlichen Lebensumstände der Familienmitglieder, u. a. homosexuelle Partnerschaft oder Charaktere mit Migrationshintergrund, im Mittelpunkt der Handlung. In beiden Serien wird zwar auch das Leben der einzelnen Kernfamilien gezeigt, im Vordergrund stehen aber der großfamiliäre Verband und die dortigen Konflikte. Ähnlich wie in Mom werden unterschiedliche Lebensphasen - das erste Date eines Pärchens, das erste Kind, langjährige Ehen - gegenübergestellt, dies wird vor allem in Life in Pieces durch die Aufteilung der Episoden in vier Abschnitte deutlich, die jeweils ein anderes Familienmitglied fokussieren. Besonders deutlich hebt Modern Family den Zusammenhalt in der Großfamilie und die gemeinsame Problemlösung hervor. Trotz eskalativer Konflikte und widerstreitender Ansichten finden die Mitglieder der Familie immer wieder zueinander (vgl. Modern Family I/1: 20:00, I/3: 19:00, I/4: 18:00). In Shameless (in Deutschland auf Prime Video verfügbar) übernimmt die älteste Tochter einer großen Familie der Unterschicht aufgrund der Verantwortungslosigkeit des arbeitslosen und alkoholabhängigen Vaters die Mutterrolle für ihre fünf Geschwister (vgl. z. B. Shameless I/1: 2:30 - hier weckt Tochter Fiona ihre Geschwister). Wiederkehrende Problematiken wie Geldmangel, ständiger Alkoholkonsum (auch der jüngeren Familienmitglieder vgl. Shameless I/1: 0:30, I/2: 
1:05) und die (erwachende) Sexualität der älteren Kinder machen das Zusammenleben der Familie, die auch Nachbarn und Bekanntschaften miteinschließt, zu einem beständigen und chaotischen Kampf. Dennoch ist der Zusammenhalt trotz aller Konflikte stark (z. B. bei der Suche nach dem verschwundenen Vater vgl. Shameless I/2: 14:00) und gelegentlich stellen sich Szenen einer gewissen familiären Harmonie ein (vgl. Shameless I/1: 56:00).

Bereits durch die oberflächliche Beschreibung dieser Formate wird deutlich, dass die Kernfamilie vielleicht ein dominanter medialer Mythos und ein verbreitetes Ideal, aber nicht so alternativlos und so ausnahmslos das Zentrum serieller Familiendarstellung ist, wie es die Video-on-Demand-Serien des Korpus nahelegen. Kann an dieser Stelle auch keine Detailanalyse erfolgen, hebt die scheinbare Heterogenität dieser TV-Serien hervor, dass ein eingehender Vergleich von Video-on-Demand-Produktionen mit Serien des konventionellen TV medienwissenschaftlich lohnenswert wäre und das Gesamtbild der medialen Familienrepräsentation bereichern würde. Das Fernsehen wird im Überschwang der Digitalisierung oft als konservatives Medium, als Teil der Vergangenheit wahrgenommen. Es ist jedoch nicht ausgeschlossen, dass sich im Zuge eines Vergleichs herausstellt, dass das Fernsehen als Produzent und Distributor jener inhaltlichen Innovation fungiert, die eigentlich den Video-on-Demand-Anbietern zugeschrieben wird. Möglicherweise sind es gerade die Video-on-Demand-Serien, die sich - unter dem Vorwand der Innovation - inhaltlich konservativen Narrativen zuwenden, um sich mit ,Bewährtem‘ im medialen Konkurrenzkampf zu behaupten, während konventionelle TV-Sender inhaltlich innovativ sind.

Ebenfalls lohnenswert wäre eine genaue Analyse internationaler Produktionen und deren Gegenüberstellung mit US-amerikanischen. Besonders deutsche Produktionen weisen - wie der Blick auf die Serien Dark und How to Sell Drugs Online (Fast) - deutliche Alleinstellungsmerkmale auf, selbst wenn sie unter dem Dach von Netflix produziert wurden. In den Weltmodellen beider Formate nimmt - wie auch in US-amerikanischen Serien - das Streben nach Freundschaft und Liebe einen hohen Stellenwert ein. Dass die gezeigten Familien durchgehend defizient sind, ist ebenso keine große Differenz zu den Formaten meines Korpus. Der Unterschied zu den US-amerikanischen Vergleichsserien besteht hier darin, dass eine ,Reparatur ' defizienter Verhältnisse nicht unbedingt angestrebt wird. Scheidung kann eine Lösung sein ebenso wie eine alleinerziehende Familie keiner, Vervollständigung ' bedarf, sondern mit nur einem Elternteil funktionieren kann. In manchen Fällen scheint es erstrebenswert, die Familie zu verlassen, um sich aus einem Kreislauf an Konflikten und Lügen zu befreien. Die Charaktere leben eher mit den bestehenden Problemen als gegen sie. Beiden Formaten ist 
zudem gemein, dass die Darstellung von Männern und Frauen bezüglich beruflicher und familiärer Autorität wesentlich egalitärer ist. In beiden Serien tauchen ebenso Polizistinnen wie Polizisten sowie Frauen und Männer in leitenden Positionen auf. Besonders im Vergleich zu den konservativeren US-amerikanischen Formaten ist eine gesellschaftliche Gleichberechtigung hier viel eher vorhanden. In einer Retrospektive in Dark wird sogar gezeigt, dass das Atomkraftwerk in den 1980er Jahren von einer Frau geleitet wurde (vgl. Dark I/3: 14:00). Selbst die deutsche Vergangenheit ist also fortschrittlicher als die Gegenwart der USA. Abweichungen von der Normalität, wie die Transsexualität eines Charakters in Dark oder die Taubstummheit eines kleinen Mädchens, werden nicht explizit thematisiert. Es findet kein direkter Fingerzeig auf die Problematik statt, sondern die Serien zeigen das Leben der Figuren mit diesen Umständen.

Diese deutschen Formate sind auffallend weniger in konservativen und traditionellen Strukturen verankert als die primären Serien meines Korpus. Der Traum von einer familiären Perfektion und einer absolut stabilen Zukunft sowie das Ideal Kernfamilie sind weniger präsent, ebenso das klare Autoritätsgefälle zwischen den Geschlechtern. Die Inszenierung zielt eher darauf ab, eine vielfältige gesellschaftliche Realität zu zeigen, als einen Mythos zu perpetuieren und als Leitbild über das gesamte Geschehen zu stellen. Weniger eine bestimmte Moral oder ein bestimmtes Pathos als vielmehr das Erzählen einer Geschichte stehen im Vordergrund.

Auch in anderen internationalen Formaten zeigen sich durchaus nationale Eigenheiten: Das Netflix Original Ingobernable, dreht sich um Skandale in der Familie des mexikanischen Präsidenten. Obwohl Familie ein bedeutsamer Handlungsimpuls der Charaktere in Ingobernable ist und sich Handeln und Gebaren der Charaktere nicht ausschlaggebend von dem US-amerikanischer Figuren unterscheidet, ergeben sich klare Unterschiede bezüglich der inhaltlichen Gewichtung und der Modellierung der Familienthematik. Die Dramatik und die Drastik der Auseinandersetzung zwischen Eheleuten finden sich auf diese Art in keinem der zuvor analysierten Beispiele. Hier bewegt sich die mexikanische Serie auf einer kulturell spezifischen Ebene der Melodramatik. Durch die Fokussierung einer tragischen und verworrenen Beziehungssituation und durch eine Frau als zentraler Charakter stellt sich Ingobernable eher in die Tradition der lateinamerikanischen Telenovela (vgl. Tufte 2015: 86, vgl. Trinta 1998: 275, vgl. Michael 2010: 207) als in die der US-Kriminal- oder Familienserie. Auch im Vergleich mit anderen internationalen Formaten zeigen sich trotz einer wachsenden Globalisierung und des zweifellos großen US-amerikanischen Einflusses weiterhin nationale Spezifika. Ein weiterer Vergleich wäre hier von großem Interesse. 
Wie - so lässt sich weiterhin fragen - verhält es sich darüber hinaus mit dem deutschen Fernsehen? Spielfilme mit Familienthematik - seien es TV, Kino oder Produktionen der Video-on-Demand-Anbieter wie The Week Of (2018; Netflix), Father of the Year (2018; Netflix), Marriage Story (2019; Netflix) oder Beautiful Boy (2018; Prime Video) - wurden ebenfalls außer Acht gelassen und verdienen eine eigenständige Analyse. Wie sich also zeigt, sind die Anknüpfungsmöglichkeiten an diese Studie, um den gezeigten Ausschnitt zu einem Gesamtbild zu ergänzen, überaus vielfältig.

Ist die Video-on-Demand-Serie bzw. sind die Serien meines Korpus nun in ihrer Gesamtheit repräsentativ für die US-amerikanische Serienlandschaft? Um dies $\mathrm{zu}$ beantworten, müsste oben genannter Vergleich mit TV-Serien angestellt werden. So wie es möglich ist, in den Produktionen des Free-TV auf innovative Inhalte zu stoßen, ist es nicht ausgeschlossen, dass letztlich auch hier eine ähnliche Angleichungsstrategie stattfindet und eine ähnliche ScheinHeterogenität vorliegt wie in den Serien des Analysekorpus. Fest steht, dass Video-on-Demand ein ausgesprochen einflussreicher Faktor im Bereich der populärkulturellen Unterhaltungsmedien und dementsprechend wirkmächtig über die Grenzen der Distributionsplattformen hinaus ist. Die Frage ist hierbei, ob eine intermediale Angleichung stattfindet oder ob TV-Serien sich bewusst abgrenzen, um ein Alleinstellungsmerkmal herauszubilden. Angesichts der bereits stattfindenden Verzahnung von TV und Streaming-Diensten - TV-Serien werden häufig international auf Video-on-Demand-Plattformen angeboten - liegt vor allem der Schluss nahe, dass es sich um ein ausgesprochen interdependentes Feld handelt, auf dem erfolgreiche, d. h. von den Rezipierenden positiv aufgenommene, Entwicklungen im einen Bereich nachahmende Entwicklungen im anderen nach sich ziehen, von denen sich andere Produktionen wiederum bewusst abgrenzen.

Ausgehend von der Familiendarstellung in der US-amerikanischen Video-onDemand-Serie zeigt sich, dass nicht nur sie sich mit Hilfe des Semiosphärenmodells beschreiben lässt. Gerade durch den hier angedeuteten Kulturvergleich wird deutlich, dass die internationale Video-on-Demand-Serien-Produktion mit Hilfe der Semiosphäre als kohärentes System modellierbar wird. Lotmans Modell mit dessen Hilfe sich die internationale Familienserie als Semiosphäre mit zahlreichen - bezüglich nationaler Herkunft und spezifischer medialer Distribution - untergeordneten Semiosphären verstehen lässt, bietet sich dementsprechend als überaus fruchtbarer Ansatz für weitere, zukünftige Vergleiche an.

$\mathrm{Zu}$ Beginn der Untersuchung wurde über den potenziell innovativen Charakter der Video-on-Demand-Serie gesprochen - ein Eindruck, der sich in Hinblick auf die Familiendarstellung als zentrales mediales Paradigma und Kern der errichteten 
Weltmodelle nicht bewahrheitet hat. Ist der Gedanke der Neuheit und der Veränderung nun lediglich oberflächliche Euphorie oder Vermarktungsinstrument? Die Antwort ist zwiespältig. So verlockend dies scheint, wäre es vorschnell und naiv, hinter jedem neuen Medium zugleich eine inhaltliche Revolution zu erwarten, und selbstverständlich ist es eine gebräuchliche Strategie durch das Versprechen der Neuheit und des noch nie Gesehenen das Interesse der Konsumierenden zu wecken. Indes handelt es sich bei Video-on-Demand-Serien um ein relativ neues Phänomen, das sich erst seit wenigen Jahren der Popularität erfreut. Was die Zukunft bringt und welchen Einfluss Streaming-Dienste auf die Medienproduktion haben, ist zum aktuellen Zeitpunkt nur vage zu erahnen. Mit dieser Studie möchte ich nicht mehr, aber vor allem nicht weniger als einen Grundstein für zukünftige Forschungen legen, die sich mit der digitalen Medienproduktion auseinandersetzen, damit, welche Veränderungen dieser so junge und dennoch bereits derart einflussreiche Kulturbereich mit sich bringen wird und damit wie dieser in Interaktion mit ,älteren` Bereichen der Medienproduktion steht.

Open Access Dieses Kapitel wird unter der Creative Commons Namensnennung 4.0 International Lizenz (http://creativecommons.org/licenses/by/4.0/deed.de) veröffentlicht, welche die Nutzung, Vervielfältigung, Bearbeitung, Verbreitung und Wiedergabe in jeglichem Medium und Format erlaubt, sofern Sie den/die ursprünglichen Autor(en) und die Quelle ordnungsgemäß nennen, einen Link zur Creative Commons Lizenz beifügen und angeben, ob Änderungen vorgenommen wurden.

Die in diesem Kapitel enthaltenen Bilder und sonstiges Drittmaterial unterliegen ebenfalls der genannten Creative Commons Lizenz, sofern sich aus der Abbildungslegende nichts anderes ergibt. Sofern das betreffende Material nicht unter der genannten Creative Commons Lizenz steht und die betreffende Handlung nicht nach gesetzlichen Vorschriften erlaubt ist, ist für die oben aufgeführten Weiterverwendungen des Materials die Einwilligung des jeweiligen Rechteinhabers einzuholen. 